\title{
Seriengründer: Erfolg macht erfolgreich
}

\author{
Korbinian Wester
}

\section{Relevanz}

Innovative Unternehmensgründungen erneuern die Wirtschaft. Aber vielen Gründern mangelt es an Kapital, an unternehmerischem Knowhow und an Erfahrung. Das Risiko ist gross, nicht alle Start-ups haben das gleiche Potenzial, und nicht jede Gründung lohnt für die Gesellschaft. Um die neuen Start-ups mit den besten Aussichten herauszufiltern und auf Erfolg zu trimmen, braucht es leistungsfähige Wagniskapitalgeber. Sie geben Kapital erst nach sorgfältiger Auswahl und leisten Beratung und Überwachung. Aber das Neue ist auf dem Markt noch nicht getestet. Das Potenzial der Start-ups ist schwer einzuschätzen. Da zählt die Erfahrung in der Vergangenheit. Einem Seriengründer, der bereits einmal Erfolg hatte und Erfahrung sammeln konnte, dem vertraut man eher. Auch ein Wagnisfinanzier, der auf einen erfolgreichen Leistungsausweis zurückblicken kann, geniesst bei den Gründern und ihren Kunden, Zulieferern und Banken mehr Vertrauen. Erfolg macht erfolgreich.

\section{Quelle}

Gompers, Paul, Anna Kovner, Josh Lerner und David Scharfstein (2010), Performance Persistence in Entrepreneurship, Journal of Financial Economics 96, $18-32$.

\footnotetext{
K. Wester $(\bowtie)$

Universität St.Gallen, St.Gallen, Schweiz

E-Mail: korbinian.wester@student.unisg.ch

(C) Der/die Autor(en) 2021

C. Keuschnigg und M. Kogler (Hrsg.), Die Wirtschaft im Wandel, 
Start-ups bringen die Wirtschaft in Schwung. Sie schaffen zusätzliche Arbeitsplätze und bringen Innovationen und neue Produkte hervor. So steigern sie den Wohlstand und vergrössern die Auswahlmöglichkeiten der Konsumenten. Gerade bei grossen wirtschaftlichen Umbrüchen wie z. B. im Zeitalter der Digitalisierung sind Innovationen notwendig, damit eine Volkswirtschaft erfolgreich bleibt. Welche Start-ups sind besonders erfolgreich?

Die Gründung und Finanzierung eines neuen Unternehmens ist mit erheblichen Risiken behaftet. Viele Start-Ups erweisen sich als nicht überlebensfähig und verschwinden nach kurzer Zeit wieder. Welche Neugründungen haben die grössten Erfolgschancen und sollen Kapital erhalten? In einer 2010 veröffentlichten Arbeit gehen Paul Gompers, Anna Kovner, Josh Lerner und David Scharfstein dieser Frage nach. Sie stellen die unternehmerische Erfahrung des Gründers in den Mittelpunkt ihrer Analyse. Manche Unternehmer werden zu Seriengründern, indem sie nach Gründung eines Start-ups ein weiteres Projekt beginnen. Dies kann unabhängig davon geschehen, ob die erste Gründung erfolgreich war oder nicht. Die Forscher untersuchen, ob solche Seriengründer erfolgreicher sind als jene Unternehmer, die zum ersten Mal ein Start-up gründen.

Die Wissenschaftler untersuchen Neugründungen in den USA zwischen 1986 und 2000, welche mit Risikokapital finanziert wurden. Ihr Datensatz enthält Informationen zu 3796 Unternehmen mit 8753 Gründern. Der Unternehmenserfolg wird daran gemessen, ob ein Start-Up bis Dezember 2007 an die Börse gegangen ist bzw. die notwendigen Unterlagen bis zu diesem Zeitpunkt eingereicht hat. Zudem zeigen die Daten, ob es sich bei einem Unternehmen um eine Seriengründung handelt, das heisst, ob mindestens einer der Gründer schon vorher über Erfahrung mit einer risikokapitalfinanzierten Gründung verfügte.

Die Anzahl der Unternehmer ist von 1980 bis 1994 langsam aber stetig angewachsen. Danach stieg sie rapide an, wobei sich die Zahl zwischen 1994 und 1995 fast verdoppelte. Die Wissenschaftler sehen darin die Auswirkungen des Internet-Booms. Insgesamt hat sich zwischen 1980 und 1990 die Zahl der Gründer, die Risikokapital erhalten haben, von 11 auf 1661 vervielfacht. Auch der Anteil von Seriengründern hat über die Jahre leicht zugenommen. Waren es im Jahr 1985 nur 7,1 \%, so hatten im Jahr 1999 bereits 9,5\% aller Gründer Erfahrung aus vorangegangenen Projekten.

Die Anzahl der erfassten Gründer hat sich zwischen 1980 und 1990 mehr als verhundertfacht. Dabei stieg der Anteil von Seriengründern von 7.1 Prozent auf 9.5 Prozent an.

Die Erfolgschancen von Unternehmensgründungen streuen stark. Im Durchschnitt schafften 25,7 \% aller Gründungen den Börsengang. Seriengründer waren 
dabei besonders erfolgreich. Ihre Erfolgswahrscheinlichkeit lag bei 36,7 \% für ihr erstes Start-up und bei 29,1 \% für nachfolgende Gründungen. Die ökonometrischen Schätzungen, die auch eine Reihe anderer Einflussgrössen berücksichtigen, zeigen ähnliche Ergebnisse. Start-Ups von Seriengründern haben eine um vier Prozentpunkte höhere Erfolgswahrscheinlichkeit als jene von Erstgründern, welche ihr Unternehmen in 20,9\% der Fälle an die Börse bringen. Die höhere Erfolgswahrscheinlichkeit im Durchschnitt ist hauptsächlich auf jene Serienunternehmer zurückzuführen, die bereits früher mit ihrem Start-up Erfolg hatten. Bei ihnen liegt die Erfolgswahrscheinlichkeit einer weiteren Neugründung bei 30,3\%. Dagegen haben Seriengründer, die in der Vergangenheit scheiterten, nur eine 21,8 \%ige Chance. Erstgründern gelingt es nur zu 20,9\%, ein Unternehmen erfolgreich aufzubauen.

Seriengründer haben eine um 4 Prozentpunkte höhere Erfolgswahrscheinlichkeit als der Durchschnitt der Gründungen. Seriengründer, die mit einem Unternehmen bereits Erfolg hatten, führen spätere Gründungen zu 30.3 Prozent zu erneutem Erfolg. Jenen, die bereits einmal scheiterten, gelingt dies nur zu 21.8 Prozent.

Die empirische Evidenz zeigt eine Beständigkeit bei erfolgreichen Unternehmensgründungen auf. Als Ursache dafür sehen die Wissenschaftler zwei Faktoren. Erfolg spiegelt zum einen die unternehmerischen Fähigkeiten wider. Diese umfassen sowohl die Managementqualitäten als auch das richtige Gespür, ein bestimmtes Produkt zur richtigen Zeit auf den Markt zu bringen. So waren beispielsweise $52 \%$ der Computerunternehmen, die 1983 gegründet wurden, erfolgreich. Zwei Jahre später war der Zeitpunkt bereits nicht mehr so günstig. Nur $18 \%$ der im Jahre 1985 gegründeten Computerunternehmen hatten Erfolg. Die unternehmerischen Fähigkeiten und das Gespür für den Markteintritt im richtigen Zeitfenster sind entscheidend für die Erfolgschancen eines Start-Ups.

Früherer Erfolg erhöht zudem das Vertrauen potenzieller Investoren, Zulieferer und Kunden. Da es für diese oft schwierig ist, die tatsächlichen Fähigkeiten eines Gründers einzuschätzen, vertrauen sie auf die unternehmerische Erfahrung, wie sie anhand vergangener Erfolge nachgewiesen ist. Seriengründern gelingt es daher eher, zusätzliche Ressourcen für ihr Start-Up aufzutreiben, was ihre Erfolgschancen neuerlich steigert. Während nur $46 \%$ der Erstgründungen in einem frühen Stadium Risikokapital aufnehmen können, sind es bei späteren Gründungen $62 \%$ der Unternehmen. Zudem erhalten Erstgründer die erste Finanzierung erst nach 37 Monaten, während erfahrene Gründer diese bereits nach 20 Monaten erhalten.

Erstgründer haben es schwerer, Risikokapital aufzutreiben. Sie warten fast doppelt so lange auf eine Risikokapitalfinanzierung wie Seriengründer. 
Eine vertrauensbildende Wirkung geht auch von der Erfahrung des Risikokapitalgebers aus, welcher das Unternehmen (mit-)finanziert. Zulieferer, weitere Investoren und Kunden vertrauen stärker auf das Urteil eines erfahrenen Risikokapitalgebers und sind dann eher bereit, ein Unternehmen $\mathrm{zu}$ unterstützen. Zählt der Risikokapitalgeber zu den $25 \%$ der erfahrensten Investoren, so ist die Erfolgswahrscheinlichkeit eines von ihm mitfinanzierten neuen Unternehmens mit $21,7 \%$ um ca. 5 Prozentpunkte höher als bei anderen Gründungen, deren Risikokapitalgeber zu den $25 \%$ der unerfahrensten Investoren gehört. Diese höhere Erfolgswahrscheinlichkeit kommt vor allem zwei Gruppen zu Gute, nämlich Erstgründern und solchen Seriengründern, die bisher nicht erfolgreich waren. Erstgründer mit einer Finanzierung von erfahrenen Risikokapitalgebern sind zu 20,9\% erfolgreich, während jene mit unerfahrenen Finanziers nur eine Chance von $14,3 \%$ haben, erfolgreich zu sein. Bei Seriengründern, bereits einmal scheiterten, ist der Unterschied mit 25,9 gegenüber 17,7 \% sogar noch grösser.

Wird ein Unternehmen von einem erfahrenen Risikokapitalgeber finanziert, so ist das Unternehmen in 21.7 Prozent der Fälle erfolgreich. Bei Finanzierung durch einen unerfahrenen Risikokapitalgeber nur in 16.5 Prozent der Fälle.

Schliesslich schätzen die Forscher, wie sich die „market timing“ Fähigkeiten eines Unternehmers, also das Gespür für den Markteintritt zur richtigen Zeit, auf die Erfolgschancen des Start-ups auswirken. Dazu messen sie den Erfolg der gesamten Branche. Hat ein Gründer sein erstes Unternehmen in einem für die Branche sehr guten Jahr gegründet, verfügt er demnach über gute „,market timing" Fähigkeiten. In diesem Fall beträgt seine Erfolgswahrscheinlichkeit bei der zweiten Gründung 30,5\%. Ein Unternehmer, der seine erste Gründung in einem branchenweit eher erfolglosen Jahr auf den Weg brachte, ist hingegen nur mit einer Wahrscheinlichkeit von 23,7\% erfolgreich. Abb. 1 fasst die Erfolgschancen von Neugründungen unter den verschiedenen Voraussetzungen zusammen.

Die Studie zeigt, dass es eine gewisse Beständigkeit in der erfolgreichen Gründung von Unternehmen gibt. Nicht nur unternehmerische Fähigkeiten, sondern auch der Erfolg in der Vergangenheit zählen. Erfolgreiche Gründer haben bessere Chancen, später ein neues Start-Up zu gründen. Das liegt zum einen daran, dass sie im Durchschnitt bessere Managementqualitäten, aber auch ein besseres Gespür für den Markteintritt zum richtigen Zeitpunkt mitbringen. Zum anderen liegt es auch daran, dass sie durch ihre Erfahrung und ihren bereits einmal bewiesenen Erfolg besseren Zugang zu Finanzierung haben und auf mehr Vertrauen bei Kunden und Zulieferern stossen. 


\section{Erfolgswahrscheinlichkeiten bei Neugründungen}

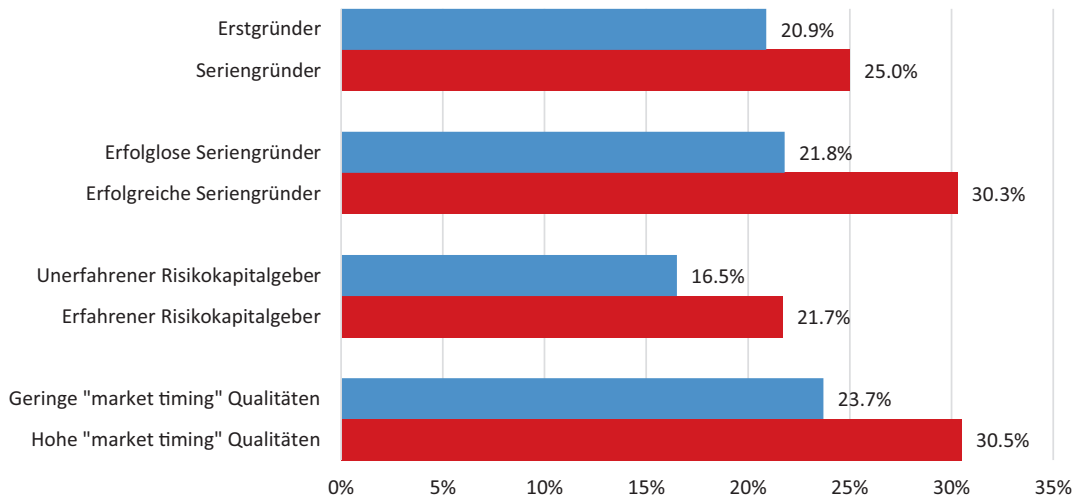

Abb. 1 Geschätzte Erfolgswahrscheinlichkeiten für Neugründungen. (Quelle: Selbst erstellte Grafik aus Zahlen von Gompers et al. 2010)

Open Access Dieses Kapitel wird unter der Creative Commons Namensnennung 4.0 International Lizenz (http://creativecommons.org/licenses/by/4.0/deed.de) veröffentlicht, welche die Nutzung, Vervielfältigung, Bearbeitung, Verbreitung und Wiedergabe in jeglichem Medium und Format erlaubt, sofern Sie den/die ursprünglichen Autor(en) und die Quelle ordnungsgemäß nennen, einen Link zur Creative Commons Lizenz beifügen und angeben, ob Änderungen vorgenommen wurden.

Die in diesem Kapitel enthaltenen Bilder und sonstiges Drittmaterial unterliegen ebenfalls der genannten Creative Commons Lizenz, sofern sich aus der Abbildungslegende nichts anderes ergibt. Sofern das betreffende Material nicht unter der genannten Creative Commons Lizenz steht und die betreffende Handlung nicht nach gesetzlichen Vorschriften erlaubt ist, ist für die oben aufgeführten Weiterverwendungen des Materials die Einwilligung des jeweiligen Rechteinhabers einzuholen.

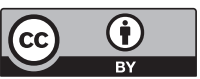

